\title{
Pour le progrès des « arts » en France : les expositions nationales des produits de l'industrie (1798-1849)
}

For the progress of the arts in France: national exhibitions of products of the industry (1798-1849)

\section{Christiane Demeulenaere-Douyère}

\section{OpenEdition Journals}

Édition électronique

URL : http://journals.openedition.org/artefact/3898

DOI : 10.4000/artefact.3898

ISSN : 2606-9245

Éditeur :

Association Artefact. Techniques histoire et sciences humaines, Presses universitaires du Midi

Édition imprimée

Date de publication : 15 octobre 2019

Pagination : 53-73

ISBN : 978-2-8107-0650-1

ISSN : 2273-0753

\section{Référence électronique}

Christiane Demeulenaere-Douyère, «Pour le progrès des « arts » en France : les expositions nationales des produits de l'industrie (1798-1849) », Artefact [En ligne], 10 | 2019, mis en ligne le 06 août 2020, consulté le 28 novembre 2020. URL : http://journals.openedition.org/artefact/3898 ; DOI : https:// doi.org/10.4000/artefact.3898

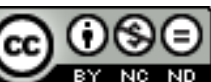

Artefact, Techniques, histoire et sciences humaines est mise à disposition selon les termes de la Licence Creative Commons Attribution - Pas d'Utilisation Commerciale - Pas de Modification 4.0 International. 


\section{Pour le progrès des «arts" en France : les expositions nationales des produits de l'industrie (1798-1849)}

\section{Christiane Demeulenaere-Douyère}

\section{Résumé}

Bien avant les grandes expositions internationales du second $x x^{e}$ siècle qui restent inscrites comme des manifestations de modernité et de splendeur, voire d'extravagance, la France organise des expositions des produits de l'industrie strictement nationales. Plus modestes et moins brillantes, elles apparaissent à la fin du XvIII siècle, justifiées par la volonté de stimuler l'industrie française affaiblie par la Révolution et le souci de réaliser un rattrapage technique par rapport à l'Angleterre. Après l'improvisation de 1798 , dix expositions de même nature se succèdent jusqu'en 1849 , d'abord avec une périodicité irrégulière, puis, à partir de 1834 , avec plus de faste et sur un rythme quinquennal maintenu, annonçant déjà les futures grandes expositions internationales. L'article présente ces diverses manifestations, en s'efforçant d'en éclairer les racines profondes, les principes, les caractéristiques et les apports, et leurs éventuels prolongements dans les expositions internationales du second $\mathrm{xIX}^{\mathrm{e}}$ siècle.

\section{Mots-clés}

agriculture, arts décoratifs, expositions industrielles, expositions internationales, industrie

99 Christiane Demeulenaere-Douyère, « Pour le progrès des "arts" en France : les expositions nationales des produits de l'industrie (1798-1849) », Artefact, 10, 2019, p. 53-73. 


\section{For the progress of the arts in France: national exhibitions of products of the industry (1798-1849)}

\section{Abstract}

Well before the major international exhibitions of the second half of the $19^{\text {th }}$ century remembered as manifestations of modernity and splendor, even extravagance, France organized strictly national exhibitions of products of the industry. More modest and less brilliant, they appeared at the end of $18^{\text {th }}$ century, driven by the desire to stimulate the French industry weakened by the Revolution and to make a technical catch-up with England. After the improvisation in 1798 , ten exhibitions of same nature succeeded each other until 1849, first with an irregular periodicity, from 1834 with more pomp and on a maintained fiveyear rhythm, announcing future major international exhibitions. This paper aims to present these various exhibitions, attempting to clarify the deep roots, the principles, the characteristics and their possible extensions in the international exhibitions of the second nineteenth century.

\section{Keywords}

agriculture, artcrafts, industry, industrial exhibitions, international exhibitions 
$\mathrm{L}$ es expositions internationales qui ont laissé une marque forte dans l'histoire du monde occidental, ne sont pas apparues soudainement, en 1851, avec la Great Exhibition of the Works of Industry of all Nations, première exposition internationale tenue à Londres, sous les verrières du Crystal Palace de Joseph Paxton (1801-1865), immense bâtisse de métal et de verre qui allait poser les canons de l'architecture expositionnaire à venir. Il convient de les réinscrire dans une chronologie plus longue, en héritières d'autres expositions aux ambitions moins spectaculaires, consacrées aux seuls produits de l'industrie nationale.

La première des expositions françaises des produits de l'industrie a lieu à l'extrême fin du XviII ${ }^{e}$ siècle, sous le Directoire, la dernière en 1849 , sous la II ${ }^{\mathrm{e}}$ République. Entre ces deux dates, se succèdent, sous le Consulat et l'Empire, trois manifestations de même nature, sur un rythme encore incertain $(1801,1802$ et 1806). Puis, après une longue suspension due aux circonstances politiques et militaires, elles reprennent sous la Restauration, en 1819 et, à partir de cette date, se suivent avec une périodicité d'abord hésitante $(1823,1827)$, puis finalement sur un rythme régulier d'une exposition tous les cinq ans (1834, 1839, 1844 et 1849).

Rapidement, le modèle français connaît le succès et se répand en Europe, d'abord dans des pays ayant appartenu à la France républicaine et impériale : Pays-Bas : 1820, 1824, 1825, 1830... ; Belgique : 1835, 1841 ; puis, dans les années 1840, dans des métropoles industrielles européennes importantes comme Mayence (1842), Berlin (1844), Vienne (1845) ou Birmingham (1849).

\section{Le concepteur : François de Neufchateau}

À l'origine est un homme politique français ${ }^{1}$, Nicolas François, dit François de Neufchateau (1750-1828), d'abord administrateur en Lorraine, puis engagé dans la Révolution dès 1791, et par ailleurs homme de lettres et agronome. Sous le Directoire, il est ministre de l'Intérieur à deux reprises, d'abord brièvement du 28 messidor au 28 fructidor an $\mathrm{V}$ [16 juillet 1797-14 septembre 1797], puis plus longuement du 29 prairial an VI au

1. Le Normand et Moléon, 1824, t. 1, p. 53 et suiv. 
4 messidor an VII [17 juin 1798-22 juin 1799], période pendant laquelle il réalise une œuvre administrative considérable, s'intéressant notamment aux questions économiques et industrielles.

Dans la Biographie intellectuelle qu' elle lui consacre, Dominique Margairaz présente un document fort éclairant ${ }^{2}$ : quelques feuillets écrits de la main de François de Neufchateau à la veille de son second passage au ministère de l'Intérieur (17 juin 1798-22 juin 1799), sur lesquels il a jeté à la hâte, en grand désordre et sans hiérarchisation, des idées de gouvernance. L'une retient particulièrement l'attention : il s'interroge sur la possibilité d' " un cabinet de l'Industrie française ou Échantillons annuels des produits les plus remarquables des fabriques de chaque département./Savoir si c'est là l'objet du Conservatoire des arts/Dans chaque département, un pareil cabinet. Honneur d'y voir ses productions admises ». Le concept même des expositions des produits de l'industrie est déjà bien présent dans son esprit.

Le ministère de l'Intérieur est alors une institution tentaculaire aux responsabilités nombreuses, dont font partie les subsistances, le commerce et l'industrie. Le second passage au ministère de François de Neufchateau correspond à un moment où sa réflexion s'attache plus particulièrement à la question de la balance du commerce, objet d'un rapport qu'il remet au Directoire le 19 thermidor an VI, puis d'un autre, le 19 nivôse an VII, sur les résultats du commerce extérieur pendant les six premiers mois de l'an VI. Pour le ministre, l'approche des questions économiques s'organise autour de deux finalités ultimes : l'amélioration des rentrées fiscales et l'accroissement des rentrées de numéraire. Pour lui, l'aisance des peuples à acquitter les contributions ne peut se soutenir sans une circulation active de numéraire. Les résultats du commerce extérieur pour l'an $\mathrm{V}$, analysés en thermidor an VI, font état d'un solde négatif de 144 millions, ce qui lui fait conclure : "Voici donc une des causes majeures de la rareté du numéraire dans la circulation ».

Le ministre souhaite ranimer l'industrie française, la tirer de la crise dans laquelle l'ont plongée les événements révolutionnaires, avec la suppression du système corporatif par la loi d'Allarde (2 et 17 mars 1791), les années sanglantes de la Terreur et les guerres révolutionnaires. Organiser une exposition peut apparaître comme un excellent moyen de relancer l'industrie nationale en promouvant ses produits auprès du grand public et aussi

2. Margairaz, 2005, p. 254-256. 
d'encourager un rattrapage technologique sur l'éternelle concurrente, la Grande-Bretagne.

Cette idée d'exposition est également soutenue dans d'autres cercles, notamment chez les " artistes-inventeurs ", dont certains finalement ont été déçus par l'institution du brevet d'invention, pourtant arrachée de haute lutte en 1791 grâce notamment à l'action déterminée de la Société des inventions et découvertes ${ }^{3}$. Mais l'obtention du brevet étant subordonnée au paiement d'une taxe, il est considéré par nombre d'inventeurs modestes comme " un nouvel impôt sur le génie ${ }^{4}$ ". À leurs yeux, une exposition publique assurerait aussi bien la publication et la protection des produits et procédés nouveaux.

Par ailleurs, la pratique des expositions se poursuit pour les beaux-arts même pendant la période révolutionnaire où le Salon de peinture et de sculpture continue de se tenir au Louvre. Selon Costaz, dans la courte histoire des expositions nationales qu'il rédige en 1806, " on pensa que les arts d'agrément ayant leur exposition, il était naturel de faire jouir les arts mécaniques du même avantage ". Accorder une exposition aux "produits de l'industrie " valorise les " arts mécaniques ", les reconnaît à l'égal des arts d'agrément, et loger l'exposition industrielle au Louvre, comme c'est le cas en 1801 et 1802, la rapproche symboliquement du Salon des beaux-arts qui s'y tient ${ }^{6}$ et par la même occasion du nouveau pouvoir.

\section{Les premières expositions des produits de l'industrie (1798-1806)}

La première exposition publique des produits de l'industrie française ${ }^{7} \mathrm{a}$ lieu, en 1798, sur le Champ-de-Mars, lieu symbolique des fêtes révolutionnaires depuis 1790. Pendant les trois derniers jours complémentaires de l'an VI [19-21 septembre 1798], elle se tient dans un local improvisé, partagé en soixante-huit arcades, disposé en carré long autour d'une

3. Demeulenaere-Douyère, 2009, p. 19-56.

4. Gaullard-Desaudray, 1792.

5. Costaz, 1806, p. 3.

6. Murga, 2017.

7. Mercier, 2018, p. 215-220. 
place au centre de laquelle s'élève très symboliquement un "Temple de l'Industrie ».

Comme l'indique la circulaire du 11 fructidor an VI, « le gouvernement doit [...] couvrir les arts utiles d'une protection particulière, et c'est dans ces vues qu'il a cru devoir lier à la fête du $1^{\text {er }}$ vendémiaire un spectacle d'un genre nouveau : l'Exposition publique des produits de l'industrie française. " Elle est donc étroitement liée à la célébration d'un événement patriotique et festif, la fête solennelle commémorant la fondation de la République, le $1^{\text {er }}$ vendémiaire an VII, pour "offrir une variété aux divertissements ${ }^{8}$.

Elle réunit cent dix exposants, la fine fleur des manufacturiers, constructeurs et fabricants, originaires majoritairement de Paris ou des environs, car l'exposition a été largement improvisée et, comme le signale le catalogue, on n'a pas eu le temps d'instruire en temps utile des dispositions du Gouvernement les départements éloignés. Sont présentées à la fois des productions prestigieuses comme celles de la Manufacture de Sèvres ou particulièrement innovantes comme " un nouvel échappement libre et à force constante » de Breguet, des balances d'essai de Lenoir, du « fer converti en acier par la simple fusion » de Jean-François Clouet, des « crayons artificiels » de Nicolas-Jacques Conté ou des « tableaux en porcelaine [...] avec des couleurs qui n'éprouvent aucun changement dans la cuisson » de Dihl et Guerhard, mais aussi nombre d'échantillons de cotons cardés et filés mécaniquement, de toile peinte, de draps, de bonneterie, de cuirs tannés et corroyés, de produits chimiques, d'outils et quincaillerie divers, etc., produits plus anodins et d'usage quotidien.

Dans les arcades 40 et 41 , " précieuse pour l'instruction publique ", sont exposés une suite complète d'étalons des poids et mesures républicains, ainsi qu'un "assortiment de mesures républicaines » et des " instructions imprimées pour faciliter l'usage de ces mesures ", réalisées par l'artiste Cicéri, dans une intention pédagogique affirmée de diffuser largement dans la population les nouvelles mesures.

Un jury de neuf membres, désignés par le ministre de l'Intérieur parmi des personnalités éminentes des milieux de la technique et de l'économie, mais dont un tiers représente aussi les arts et lettres, est chargé d'examiner les

8. Costaz, 1806, p. 3. 
ouvrages exposés et de décerner des récompenses. Douze " artistes " sont ainsi distingués et beaucoup d'autres signalés?

Visiblement séduit par l'initiative, le jury conclut son rapport par un bilan positif :

Le jury doit au gouvernement de lui déclarer que les progrès de l'industrie se lient essentiellement au maintien de l'institution qu'il vient de former. Il peut lui annoncer que le moment est arrivé où la France va échapper à la servitude de l'industrie de ses voisins ; que, partout, les arts associés aux lumières se dégagent de cette honteuse routine, qui est le caractère de l'esclavage ; que l'émulation la plus brûlante embrase toutes les têtes des artistes, et que le gouvernement n'a qu’à vouloir, pour porter les arts au degré de supériorité où s'est placée la grande nation parmi les peuples de l'Europe ${ }^{10}$.

Le ministre se saisit du succès de cette première opération - largement improvisée mais qu'il qualifie, avec emphase et cependant assez de clairvoyance, de "première pierre d'un édifice immense [...] qui s'embellira, chaque année, par les efforts conjugués du commerce et de l'industrie »-, pour confirmer le principe d'une exposition annuelle des produits de l'industrie nationale. L'affaire est politique ; le Gouvernement doit convaincre de l'intérêt qu'il porte aux "travaux des arts ", encourager l'émulation technique et contribuer au développement général des fabriques. Telles sont les missions assignées à ces expositions qui s'inscrivent aussi dans le contexte de la rivalité économique opposant la France et l'Angleterre, comme le souligne lui-même le ministre : cette exposition "n'a pas été très nombreuse, mais c'est une première campagne et cette campagne est désastreuse pour l'industrie anglaise. Nos manufactures sont les arsenaux d'où doivent sortir les armes les plus funestes à la puissance britannique ${ }^{11}$ ».

La formule conquiert sa légitimité auprès des manufacturiers et des artisans et est reprise et développée à leur demande. Sous le Consulat, deux autres expositions ont lieu. D'abord, en $1801^{12}$, dans la Cour du Louvre (Fig. 1), sous des portiques qui accueillent, pendant six jours, 229 exposants qui se partageront quatre-vingts récompenses. "On y vit figurer tout ce que

9. Exposition publique des produits de l'industrie, vendémiaire an VII, passim.

10. Exposition publique des produits de l'industrie, vendémiaire an VI, p. 25-26.

11. Flachat, 1834, p. 18. «Expositions des produits de l'industrie », p. 1157.

12. Mercier, 2018, p. 264-266. 
les arts mécaniques produisent de plus utile, et de l'exécution la plus achevée $^{13}$ ». Puis un nouveau concours s'ouvre en 1802, toujours pendant une semaine, toujours dans la Cour du Louvre, qui réunit 540 exposants et distribue 244 récompenses.

Malgré la décision de Chaptal d'organiser une exposition industrielle tous les ans (1801), l'Empire, assailli par d'autres problèmes, n'organise qu'une seule exposition à la faveur d'un bref intermède de paix continentale. Par ailleurs, il souhaite aussi, non point proposer un bazar de produits divers, mais y faire constater les progrès réalisés par l'industrie et organiser des enquêtes sur des questions touchant le développement de la production et le sort des producteurs, ce qui suggère des intervalles plus longs entre deux expositions. C'est en 1806, en marge des célébrations de la victoire d'Austerlitz, qu'il convoque une nouvelle exposition des produits de l'industrie pendant vingt-quatre jours (ouverture le 26 septembre 1806) ${ }^{14}$, cette fois sur l'esplanade des Invalides, avec 1422 exposants qui se partagent 610 récompenses. L'épais catalogue ${ }^{15}$, signé par Claude-Anthelme Costaz (1769-1858), l'éminent chef du bureau des arts et manufactures du ministère de l'Intérieur, détaille, sur plus de trois cents pages, les participations venues de l'ensemble des départements français, y compris des territoires annexés à l'Empire, avec beaucoup d'observations intéressantes et détaillées à la fois sur les caractéristiques et la qualité des produits et aussi sur les processus de fabrication ${ }^{16}$.

1806, c'est l'exposition où, entre autres, Nicolas Appert présente sa méthode de conservation des aliments, et Jean-Baptiste Launay un modèle de coupole pour la halle au blé de Paris et deux ponts de fonte de fer pour la capitale, et où on découvre un superbe papier peint panoramique intitulé «Sauvages des mers du Sud».

13. Costaz, 1806, p. 4.

14. L'Exposition industrielle de 1806 s'inscrit entre la prohibition en France des toiles en coton en provenance de Grande-Bretagne (22 février 1806) et la taxation des denrées coloniales (4 mars $1806)$ et le décret de Berlin instaurant le Blocus continental (21 novembre 1806).

15. Costaz, 1806.

16. Ce catalogue, auquel il manque malheureusement un index, fournit un tableau très documenté de l'état de l'industrie européenne en 1806. 


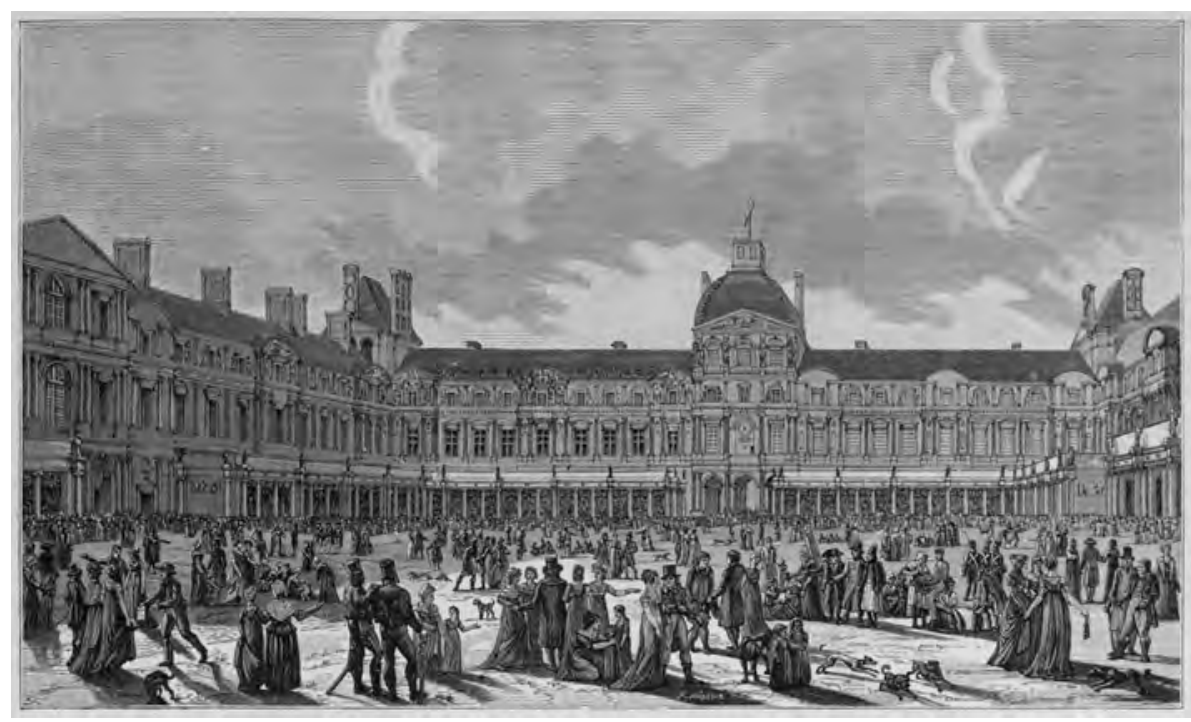

Fig. 1. - « Exposition des produits de l'industrie française, dans la cour du Louvre, 1801 »

Extrait de Fedor Hoffbauer, Paris à travers les âges. Aspects successifs des monuments et quartiers historiques de Paris depuis le XIII siècle jusqu'à nos jours, Paris, Firmin-Didot, 1875-1882, vol. 1.

\section{Sous la Restauration}

De retour au pouvoir, la Monarchie reprend à son compte l'initiative des régimes précédents de présenter, à intervalles réguliers, un panorama des productions des différentes branches de l'industrie nationale, afin de soutenir l'économie, de susciter l'émulation et de lutter contre la concurrence des nations étrangères. Et ceci d'autant plus que, d'exposition en exposition, les industriels et manufacturiers se sont pris au jeu. Exprimant souvent «le vœu du renouvellement périodique de ces solennités où sont couronnés ceux qui ont agrandi le domaine de l'industrie ${ }^{17}$ ", ils ont compris les avantages qu'ils pouvaient tirer de cette vitrine officielle sponsorisée par le pouvoir, à caractère labellisant par l'attribution de récompenses.

17. Costaz, 1819, « Avant-propos ». 
C'est ainsi que, par l'ordonnance du 13 janvier 1819, Louis XVIII adopte le principe d'une exposition publique des produits de l'industrie française au moins tous les quatre ans, à l'initiative du monarque. La prochaine est fixée en 1819, la suivante en 1821. L'exposition de 1819 ouvrira le 25 août, jour de la fête du $\operatorname{Roi}^{18}$ - on est bien dans la même logique "honorifique " qu'en 1798. Également honorifique est l'installation de l'exposition industrielle dans les salles et galeries du Louvre, inaugurant ainsi les nouveaux aménagements que vient de réaliser l'architecte Fontaine, dans les salles du rez-de-chaussée de la Colonnade, les deux escaliers et, au premier étage, les ailes de la Colonnade, du Bord de l'Eau et de l'Horloge. On peut voir dans cette attention du souverain la démonstration de l'intérêt tout particulier dont il entend honorer les arts mécaniques.

La participation des manufacturiers et des fabricants se fera sur inscription auprès de la préfecture du département de résidence. Le préfet désignera un jury local de cinq membres, chargé de statuer sur l'admission ou le rejet des objets proposés. À Paris, un jury central de quinze membres jugera les produits exposés et attribuera les récompenses (prix - médailles d'or, d'argent ou de bronze - ou mentions honorables).

L'article 7 de l'ordonnance du 13 janvier 1819 rappelle : «Un échantillon de chacune des productions désignées par le jury sera déposé au conservatoire des arts et métiers, avec une inscription particulière qui rappellera le nom du manufacturier ou du fabricant qui en sera l'auteur. » Ainsi est réaffirmé - car cette obligation figurait déjà dans la circulaire de Neufchateau d'octobre $1798^{19}$ - le lien privilégié établi entre le Conservatoire et les expositions des produits de l'industrie et le projet de constituer " pour l'industrie des archives précieuses, qui permettront de suivre les progrès, de signaler les époques stationnaires ou rétrogrades, [d'établir] une surveillance dont le résultat sera de rendre difficiles, impossibles même, ces moments de décadence qui succèdent quelquefois aux époques les plus brillantes ${ }^{20} »$.

18. Costaz, 1819, p. 379-381.

19. Circulaire du 24 vendémiaire an VII [15 octobre 1798] : «Un échantillon de chacun des produits qui auront mérité [une médaille], sera déposé au Conservatoire des arts et métiers, dans une salle consacrée à recevoir les monuments de l'exposition de chaque année. Une inscription particulière sera attachée à chaque échantillon. ", citée dans Mercier, 2018, p. 219.

20. Notice des produits de l'industrie française..., 1834, p. V. 
En 1819, la mise en œuvre de l'exposition est soigneusement organisée selon le processus défini par le texte du 13 janvier 1819. Une circulaire du 26 janvier 1819 mobilise les préfets, détermine les travaux des jurys de sélection dans les départements et règle l'organisation des envois vers Paris ${ }^{21}$. Une orientation nouvelle de la manifestation prévoit que seront attribués sur proposition du jury central non seulement des prix, en nombre non limité, mais qu'encore seront distingués, notamment par l'attribution de décorations et leur présentation au souverain, " ceux des manufacturiers ou fabricants désignés pour des prix, et qui en ayant obtenu dans les précédents concours, ou ayant, par des procédés nouveaux ou des découvertes importantes, fait faire un pas notable à l'industrie nationale, paraitront mériter un témoignage plus éclatant de la satisfaction royale ». En 1819, est réaffirmé le système des récompenses, qui existait depuis 1798 et sera ensuite assidûment appliqué dans les grandes expositions d'après 1850 .

Treize années donc après l'exposition de 1806, se tient la cinquième édition de l'Exposition publique des produits de l'industrie, pendant trente jours (25 août-23 septembre 1819), au Louvre ${ }^{22}$. Le roi vient en personne la visiter seul le 28 août et, accompagné des ministres Decazes (Intérieur) et Pradel (Maison du Roi), le 17 septembre, et la famille royale y vient plusieurs fois. On décompte 1662 exposants et les 669 récompenses sont remises, le 25 septembre, aux industriels les plus remarqués.

Le jury, qui comprend quinze membres, tous personnalités éminentes (Fontaine, Ternaux, Chaptal, Breguet, Gérard, Berthollet, etc.), publie un Rapport du Jury, dont le rédacteur est le baron Louis Costaz (1767-1842). Ce document donne, en plus de cinq cents pages, section par section, une vue d'ensemble assez précise des pièces exposées, et un chapitre recense les exposants distingués, avec un commentaire assez détaillé sur les mérites de chacun.

La Restauration manque le rendez-vous de 1821, semble-t-il, sous la pression des manufacturiers eux-mêmes qui désiraient plus de temps pour faire évoluer leurs produits.

Finalement, ce sera 1823 ! Le 19 janvier 1823, le même Louis XVIII signe une ordonnance fixant l'ouverture d'une nouvelle exposition des produits 
de l'industrie au 25 août de l'année 1823 (jour de la Fête du Roi). Les procédures d'organisation sont identiques en tout point à celles de 1819 et l'exposition est installée au Louvre, tout comme en 1819. Elle y occupe le rez-de-chaussée de la Colonnade et tout le premier étage, autour de la Cour carrée, sauf la salle du Trône et la chapelle, soit une enfilade de quarante et une salles. Ce n'est plus Fontaine mais le jeune architecte Jules de Joly ${ }^{23}$ qu'on charge des aménagements nécessaires pour accueillir les produits.

L'exposition reste ouverte durant cinquante jours (25 août-13 octobre). Y participent 1648 exposants, avec moins d'objets provenant de la province, semble-t-il, mais ceux de Paris sont très abondants. Les 1091 récompenses, plus nombreuses qu'en 1819, sont remises par le Roi dans son Cabinet des Tuileries, le 24 octobre 1823 . Le Jury central conclut son rapport, avec cette autosatisfaction qui sera si coutumière dans les expositions : «l'Exposition de 1823 fera époque dans les annales de l'industrie française ; elle a surpassé toutes celles qui l'ont précédée, tant par le nombre que l'importance des objets qu'elle a fait paraître aux yeux du public. » Il y voit une "belle fête manufacturière " ${ }^{24}$.

L'exposition suivante (la septième depuis 1798) est au rendez-vous de 1827. Une forme de régularité s'institutionnalise. Elle se tient toujours au Louvre, mais, la place y manquant à cause de la création de plusieurs galeries muséographiques dans le palais, tous les exposants ne peuvent y trouver place. Cette fois, elle ouvre ses portes le $1^{\text {er }}$ août 1827 et dure jusqu' au $1^{\text {er }}$ octobre (62 jours). On décompte 1695 exposants et 1254 récompenses. Le luxe y domine très largement sur les produits du quotidien.

\section{La Monarchie de Juillet}

La révolution de 1830 éclate au moment où l'on envisage une nouvelle exposition. La Monarchie de Juillet reprend l'idée, mais préfère ajourner l'événement jusqu'à un moment plus favorable où les manufacturiers, très

23. Architecte de la Chambre des Députés en 1821, Jules de Joly (1788-1865) devient, en 1823, également architecte du ministère de l'Instruction publique et de celui du Commerce et des Travaux publics, en 1831 ; de 1827 à 1829, il est architecte des Bâtiments civils et intervient sur les bâtiments du ministère de l'Intérieur, et travaille aussi sur l'Observatoire vers 1840 .

24. Catalogue des produits de l'industrie française admis à l'exposition publique..., 1823 ; Corbière, 1824, p. 1. 
touchés par les événements de 1830, seront à nouveau en état de montrer leurs plus belles productions.

C'est par une ordonnance du 4 octobre 1833 que le nouveau monarque, Louis-Philippe, décide que des expositions des produits de l'industrie auront lieu sur un rythme quinquennal très régulier : 1834, 1839, 1844 et $1849^{25}$.

Cependant, dès 1834, a lieu une rupture notable avec les usages précédents. Dès lors, les expositions abandonnent le Louvre, dont l'étroitesse avait déjà gêné l'organisation en 1827, pour s'installer place de la Concorde, en bas des Champs-Élysées (Fig. 2). Au centre de la place, on dresse un simulacre de l'obélisque de Louxor $^{26}$, autour duquel Louis Moreau ${ }^{27}$, un élève de Charles Percier, édifie quatre pavillons provisoires dont la superficie excède d'un tiers au moins les locaux de l'Exposition de 1827 au Louvre. D'une architecture très simple, il s'agit d'une galerie divisée en nefs par des colonnes, entourant une cour couverte ; au centre de la nef centrale, se trouvent des tables protégées par des barrières. Sur la façade, au-dessus des portes, des inscriptions et allégories précisent la destination de chaque bâtiment : machines, outils, métaux ; produits chimiques, typographie ; laine, soie, coton ; bronze, orfèvrerie, ébénisterie ${ }^{28}$.

L'exposition de 1834 reste ouverte pendant soixante jours ( $1^{\text {er }}$ mai-29 juin). Parmi les 2447 exposants, l'industrie parisienne brille particulièrement ; 1785 récompenses sont distribuées. Le Roi et la famille royale témoignent d'un intérêt marqué, par des visites et achats nombreux répartis entre les divers fabricants.

Le jury central s'étoffe. Il comporte maintenant huit commissions et produit un énorme rapport en trois volumes, très développés.

25. Bulletin des lois, $1833,2^{\mathrm{e}}$ partie, $1^{\mathrm{re}}$ section, t. VII, $\mathrm{n}^{\circ} 259$, p. 383-384.

26. L'obélisque original, offert par le vice-roi d'Égypte Méhémet-Ali à la France en 1830, ne sera placé sur la place de la Concorde que le 25 octobre 1836.

27. Louis Moreau (1790-1862) est, de 1827 à 1830, architecte du département de la Haute-Saône où il dessine de nombreux lavoirs, églises et fontaines, puis commissaire-voyer d'arrondissement à Paris de 1832 à 1858. Il est le père du peintre Gustave Moreau.

28. Notice des produits de l'industrie française..., 1834. 


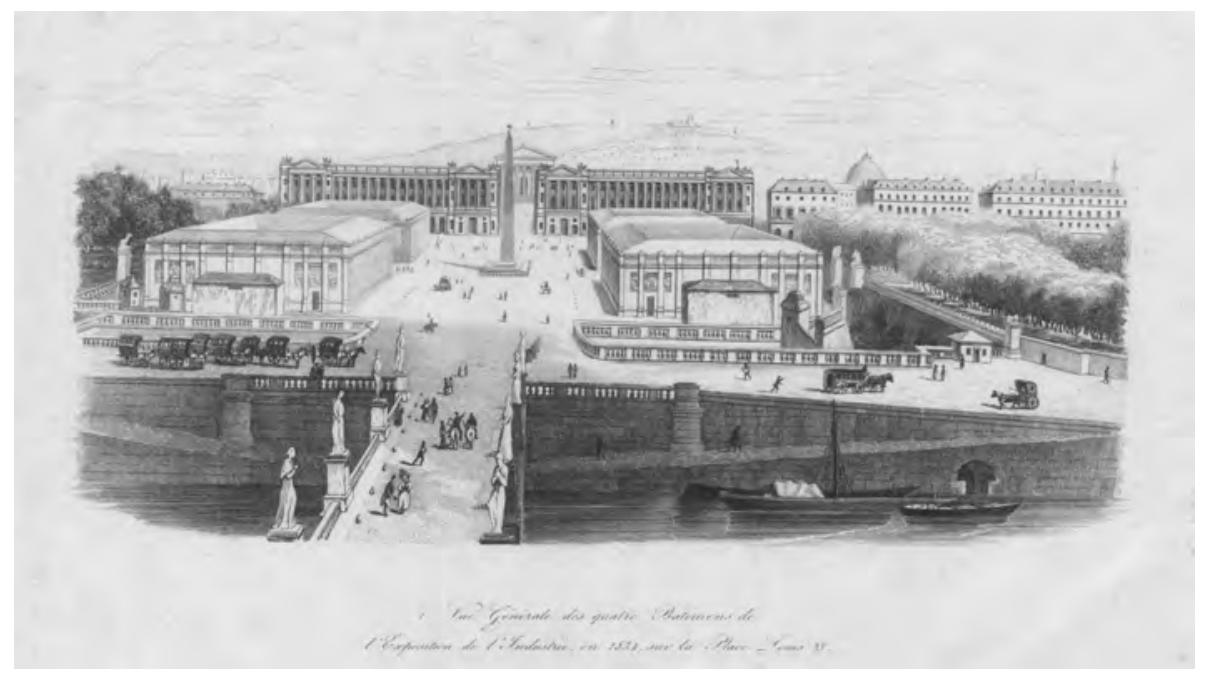

Fig. 2. - Gérard, « Vue générale des quatre bâtiments de l'exposition de l'industrie, en 1834, sur la place Louis XV »

Extrait de Stéphane Flachat, Industrie. Exposition de 1834, Paris, L. Tenré, Henri Dupuy, 1834, pl. I.

Suit l'exposition de 1839, décidée par l'ordonnance du 27 décembre $1838^{29}$. Inaugurée par le roi le 30 avril 1839, elle ouvre le lendemain dans une certaine confusion et fermera le $1^{\text {er }}$ juillet. Louis Moreau a édifié des bâtiments provisoires dans le grand carré des Champs-Élysées : la façade est constituée d'une grande galerie très simple, parallèle aux Champs-Élysées ; perpendiculairement s'ouvrent six grandes salles de $69 \mathrm{~m}$ de long sur $26 \mathrm{de}$ large. Ces bâtiments sont insuffisants pour contenir les 3281 exposants, si bien qu'il faut rajouter à la hâte d'autres salles et tout n'est pas prêt pour l'ouverture.

Le Jury central, toujours constitué de huit commissions spéciales, fournit un rapport de plus en plus détaillé. Pour la première fois, chaque partie du rapport est précédée des noms des membres de la commission chargée de sa rédaction et parait sous la signature du rapporteur. Mais de nombreuses critiques commencent à se faire entendre contre le principe de ces

29. Exposition des produits de l'industrie française en 1839..., 1839, t. 1, ordonnance du 27 décembre 1838, p. vii-viii ; circulaires, p. ix-xxv. 
expositions générales et périodiques, dans lesquelles beaucoup ne voient plus qu'un grand bazar sans intérêt.

L'exposition suivante se tient du $1^{\text {er }}$ mai au 30 juin 1844, toujours dans le grand carré des Champs-Élysées. Comme en 1839, Moreau y élève un "Palais de l'Industrie " - c'est la première fois qu'on utilise cette dénomination - en fait une construction éphémère qui subsistera jusqu'en 1853. Quatre galeries, ouvrant par une porte en façade, délimitent un vaste quadrilatère. Au nord, sont les objets de luxe (bronze, orfèvrerie, meubles, tapis, papiers peints), la lithographie, les instruments de précision ; au sud, les tissus en laine, soie, fil, coton ; à l'est, la poterie, les cristaux, la porcelaine ; à l'ouest, les instruments de musique et divers objets. Le centre, couvert contrairement à 1839 , est réservé aux machines, et le pourtour des bâtiments à l'extérieur, aux bestiaux et aux instruments agricoles ${ }^{30}$.

C'est une exposition particulièrement brillante que proposent les 3960 exposants retenus ; le 29 juillet 1844, on remet les très nombreuses (3 253) récompenses.

Le 22 novembre 1848, l'Assemblée nationale vote le principe d'une exposition des produits de l'industrie pour 1849. Ce qui fait son originalité, c'est que, pour la première fois, les produits de l'agriculture sont associés à ceux de l'industrie manufacturière dans le titre même de l'exposition : "Exposition des produits de l'industrie agricole et manufacturière ". Déjà se profile le modèle des expositions universelles qui se développeront à partir de 1855.

La période est difficile sur le plan économique et social, et organiser une telle exposition peut relever du défi. Néanmoins elle s'ouvre le $1^{\text {er }}$ juin 1849 , dans le grand carré des Champs-Élysées. Moreau est à nouveau chargé de réaliser le bâtiment : la façade est scandée par huit frontons, ornés chacun de deux génies des arts et de leurs attributs et d'inscriptions détaillant le contenu de l'exposition ; après un péristyle orné d'une mosaïque d'asphalte, on trouve une immense galerie circulaire, divisée par deux rangées de pilastres, faisant le tour d'un vaste hangar et de trois cours. À l'intérieur, sur les pans coupés du plafond à caissons, est rappelé en lettres d'or sur fond bleu le nom de plus de mille localités connues pour leur industrie ${ }^{31}$ (Fig. 3 et 4 ).

30. Dion-Tenenbaum, 1991a.

31. Dion-Tenenbaum, 1991b. 


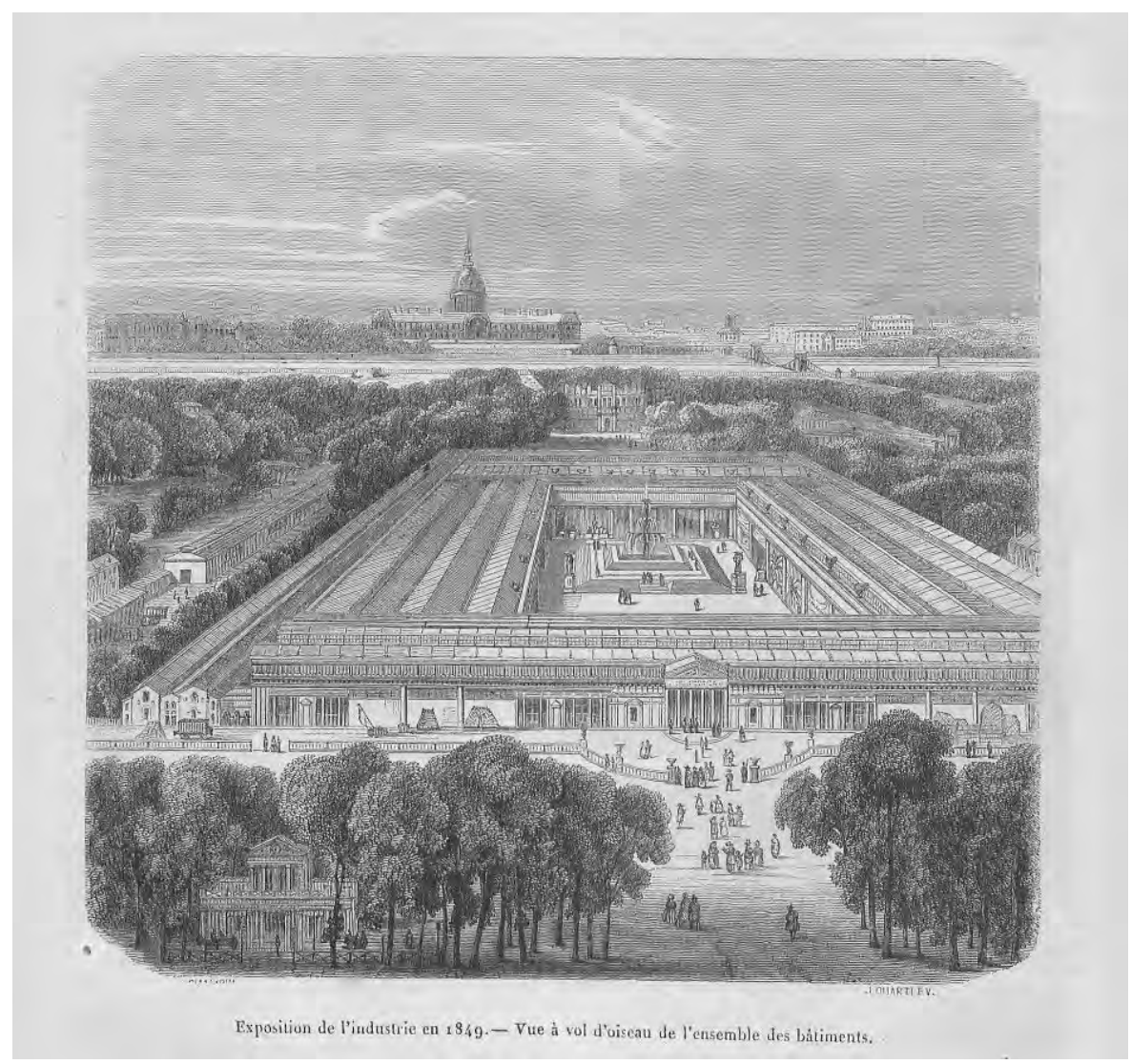

Fig. 3. - Jean-Jacques Champin, « Exposition de l'industrie de 1849. Vue à vol d'oiseau de l'ensemble des bâtiments " gravé par John Quartley

Extrait de : Le Magasin pittoresque, $17^{\mathrm{e}}$ année, 1849, p. 241. Coll. part.

Cliché Guy Lambert

Les exposants sont venus encore plus nombreux (5 494). Le jury s'accroît : soixante-cinq membres, répartis en dix commissions. La remise des récompenses a lieu le 11 novembre 1849, au Palais de Justice, en présence du nouveau président de la République, Louis Napoléon Bonaparte ${ }^{32}$.

32. Il ne faut pas oublier que Napoléon III sera le promoteur de deux expositions universelles à Paris, en 1855 et 1867 ; son expérience de 1849 n’y est sans doute pas étrangère. 


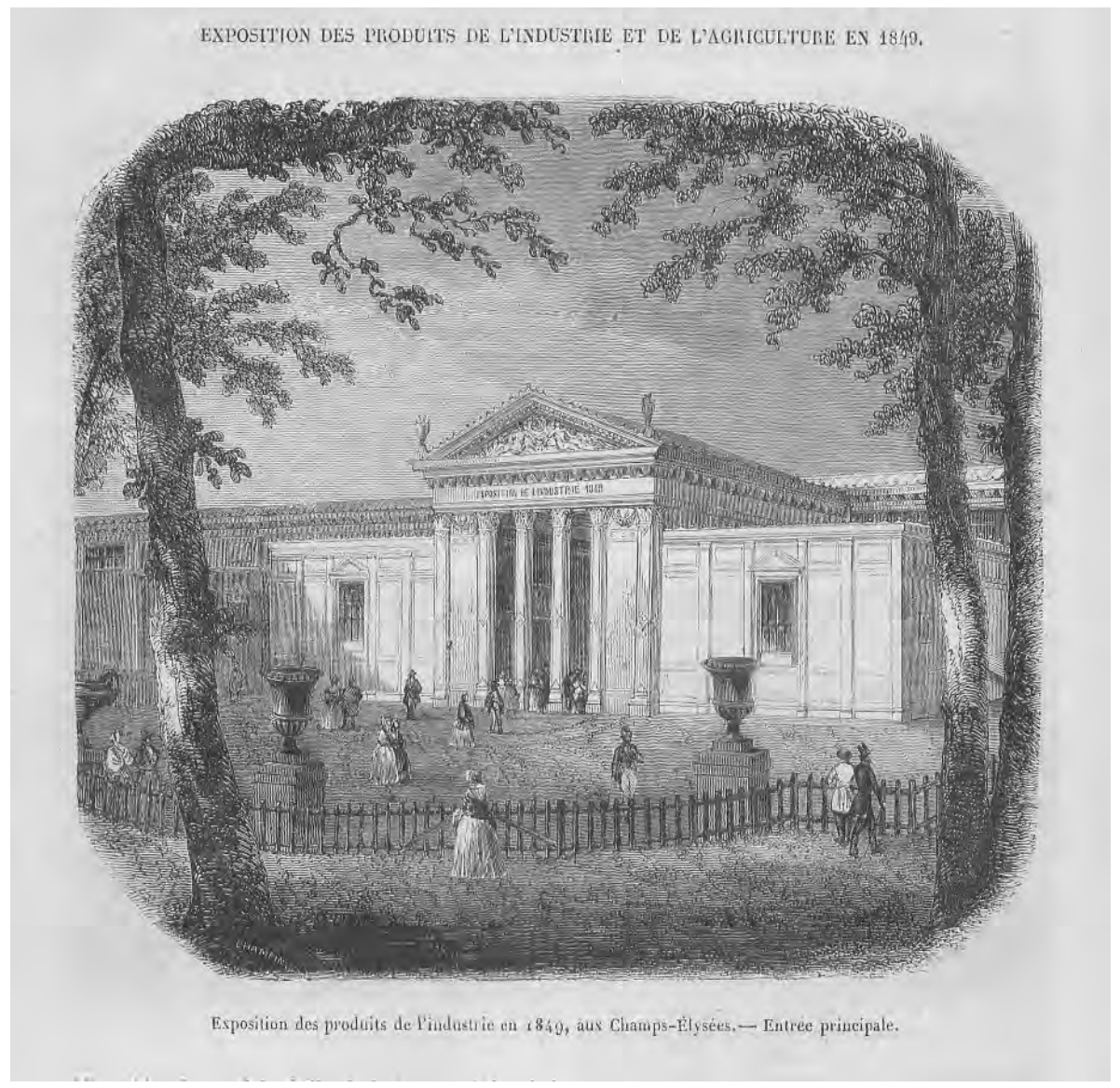

Fig. 4. - Jean-Jacques Champin, « Exposition de l'industrie en 1849. Entrée principale »

Extrait de : Le Magasin pittoresque, $17^{\mathrm{e}}$ année, 1849, p. 241. Coll. part.

Cliché Guy Lambert

\section{Un laboratoire des " grandes " expositions internationales}

Entre la très limitée exposition de 1798 qui ne rassemblait qu'une centaine d'exposants pendant seulement trois jours, et celle de 1849 qui en accueille près de six mille pendant deux mois et qui présente déjà presque tous les caractères majeurs des grandes expositions du second $\mathrm{xIX}^{\mathrm{e}}$ siècle (à l'exception, bien sûr, du caractère international), seulement un demi-siècle 
s'est écoulé ; brève durée de temps, mais radicale évolution du modèle, malgré les vicissitudes politiques et économiques que connaît cette première moitié du XIX ${ }^{\mathrm{e}}$ siècle.

L'accroissement régulier de la durée des expositions, des surfaces qui leur sont consacrées, du nombre des exposants et des récompenses décernées atteste de leur succès, même si nous manquons de statistiques officielles sur leur fréquentation par le grand public et d'informations sur leur réception par celui-ci. De même, nous voyons, peu à peu, au fil des années, se mettre en place nombre de pratiques qui seront spécifiques aux grandes expositions d'après 1851 : sélection des exposants localement par un jury départemental, rédaction d'un catalogue de plus en plus détaillé, rôle important du jury national et de son Rapport, distribution généreuse de récompenses et distinctions honorifiques, édification d'architectures éphémères... En ce sens, on peut considérer les expositions nationales comme des laboratoires d'essai où, peu à peu, souvent dans l'improvisation, mais avec pragmatisme, s'élabore la formule " exposition » et d'où sortent en ligne droite les grandes expositions internationales de la période suivante ; seule l'échelle change... Jusqu'à la localisation des expositions dans Paris : Champ de Mars, esplanade des Invalides, bas des Champs-Élysées, autant de lieux que reviendront fréquenter assidûment les expositions internationales à partir de 1855.

Quant au mot "Industrie ", il est employé ici dans une acception très large qui dépasse la seule production manufacturière mais inclut aussi l'agriculture et l'élevage, comme le souligne le titre de l'exposition de 1849, "...produits de l'industrie agricole et manufacturière ". Il traduit une vision " inclusive " de l'industrie comprise comme l'ensemble des activités humaines, de tout ce qui est utile à l'homme, et peut être lu déjà comme une préfiguration de l'" universalité " qui va s'imposer quelques années plus tard.

On peut regretter qu'il existe si peu de travaux synthétiques sur l'histoire des premières expositions des produits de l'industrie. Leurs catalogues, maintenant facilement accessibles en ligne ${ }^{33}$, constituent des mines d'informations, parfois utilisées par les historiens de l'art, surtout pour les arts

33. Notamment sur le site cnum.cnam.fr, onglet "Expositions universelles " (474 titres en ligne actuellement) et dans Gallica ; voir également Plinval de Guillebon, 2006. En revanche, on dispose aux Archives nationales seulement d'un petit nombre de documents d'archives concernant 
décoratifs, pour étudier de façon ponctuelle la production d'un artiste ou le cheminement d'une œuvre, mais rarement exploitées globalement, notamment par les historiens de l'économie et de la vie matérielle qui pourraient $\mathrm{y}$ trouver des pistes de réflexion. On dispose d'un précieux catalogue d'exposition, Un âge d'Or des arts décoratifs, 1814-1848 ${ }^{34}$, dont les rédacteurs se sont beaucoup appuyés sur cette documentation.

Mais l'ambition politique de ces expositions ne peut se résumer à montrer seulement des pièces de bijouterie et d'orfèvrerie somptueuses, des meubles précieux, des bronzes ciselés et dorés... même si, sous la Restauration notamment, on peut avoir l'impression que le but de l'exercice est de présenter des pièces de prestige que le monarque ne saurait manquer d'acheter. Il paraît utile de reprendre cette énorme masse documentaire et de l'étudier à la fois globalement et plus finement pour mieux préciser les missions de ces expositions et en analyser les caractéristiques et les évolutions. Une étude plus spécifique par branches d'industrie permettrait aussi d'en évaluer le réel impact sur le développement des « arts » et de l'économie française et une éventuelle comparaison avec des pays étrangers qui ont aussi organisé des expositions industrielles nationales serait pleine d'intérêt.

\section{Bibliographie}

Bulletin des lois, 1833.

Catalogue des produits de l'industrie française admis à l'exposition publique dans le Palais du Louvre, contenant les noms et les demeures des artistes qui les présentent..., Imprimerie Anthelme Boucher, Paris, 1823.

CorbiÈre Comte, Rapport sur les produits de l'industrie française présenté au nom du jury central..., Imprimerie royale, Paris, 1824.

Costaz Claude-Anthelme, Notices sur les objets envoyés à l'exposition des produits de l'industrie française..., Imprimerie impériale, Paris, 1806.

Costaz Louis, Rapport du Jury central sur les produits de l'industrie française..., Imprimerie royale, Paris, 1819.

Demeulenaere-Douyère Christiane, "Inventeurs en Révolution : la Société des inventions et découvertes ", Documents pour l'histoire des techniques, $\mathrm{n}^{\circ} 17$, $1^{\text {er }}$ sem. 2009, p. 19-56, en ligne : http://dht.revues.org/483.

l'organisation de ces expositions, quelques articles très lacunaires cotés F/12/501/B, 985 à 991, 5005/A et $11849^{*}$ (an IV à 1849).

34. Un âge d'or des arts décoratifs, 1814-1848, 1991. 
Dion-Tenenbaum Anne, "L'exposition des produits de l'industrie ", dans Un agge d'or des arts décoratifs, 1814-1848, Réunion des musées nationaux, Paris, 1991 a, p. 422-423.

Dion-Tenenbaum Anne, "L'exposition des produits de l'agriculture et de l'industrie ", dans Un âge d'or des arts décoratifs, 1814-1848, Réunion des musées nationaux, Paris, 1991 b, p. 472.

"Expositions des produits de l'industrie ", dans Dictionnaire universel théorique et pratique, du commerce et de la navigation..., t. I, A-G, p. 1156-1164, Paris, 1859-1861 (en ligne sur Gallica).

Exposition publique des produits de l'industrie. Catalogue des produits industriels qui ont été exposés au Champ de Mars pendant les trois derniers jours complémentaires de l'An VI..., Imprimerie de la République, Paris, vendémiaire an VII.

Exposition des produits de l'industrie française en 1839. Rapport du jury central, L. Bouchard-Huzard, Paris, 1839.

FLACHAT Stéphane, L'industrie. Recueil de traités élémentaires sur l'industrie française et étrangère. Exposition des produits de l'industrie de 1834, H. Tenré et L. Dupuy, Paris, 1834.

[Gaullard-Desaudray Charles-Emmanuel], Constitution nouvelle des sciences et des arts, envoyée par la Société du Point central des arts et métiers à L'assemblée législative, 1792, cité dans HILAIRE-PÉrez Liliane, L'invention technique au Siècle des Lumières, Albin Michel, coll. Lévolution de l'humanité, Paris, p. 399, note 128 .

Le Normand L.-Séb. et Moléon J. G. V. de, Description des expositions des produits de l'industrie française faites à Paris depuis leur origine jusqu'à celle des 1819 inclusivement..., Bachelier, Paris, 1824.

Margairaz Dominique, François de Neufchateau. Biographie intellectuelle, Publications de la Sorbonne, Paris, 2005.

Mercier Alain, Le Conservatoire des arts et métiers des origines à la fin de la Restauration (1794-1830), Musée des Arts et Métiers-Le Cnam - Éditions Snoeck, Paris-Gand, 2018.

Murga Camilla, "The crafty link: fine arts and industrial exhibitions under the Consulate and Empire ", dans Coquery Natacha, Ebeling Jörg, Perrin Khelissa Anne et SÉnÉCHal Philippe (dir.), "Les progrès de l'industrie perfectionnée ". Luxe, arts décoratifs et innovation de la Révolution française au Premier Empire, Presses universitaires du Midi, coll. Tempus Artis, Toulouse, 2017, p. 45-51.

Notice des produits de l'industrie française : Précédée d'un Historique des expositions antérieures et d'un coup d'oil général sur l'exposition actuelle, Everat, Paris, 1834. 
Plinval de Guillebon Régine de, Bibliographie analytique des expositions industrielles et commerciales en France depuis l'origine jusqu'à 1867, l'Échelle de Jacob, Dijon, 2006.

Un âge d'or des arts décoratifs, 1814-1848, [exposition], Galeries nationales du Grand Palais, Paris, 10 octobre-30 décembre 1991 [organisée par la] Réunion des musées nationaux, Réunion des musées nationaux, Paris, 1991.

\section{L'autrice}

Conservateur général du patrimoine (h), membre correspondant du Centre Alexandre-Koyré, Christiane Demeulenaere-Douyère est spécialiste de l'histoire des expositions internationales. Elle a notamment dirigé les ouvrages suivants, avec Carre Anne-Laure, Corcy Marie-Sophie et Hilaire-Pérez Liliane, Les expositions universelles en France au xix siècle. Techniques. Publics. Patrimoines, CNRS Éditions, Collection Alpha, Paris, 2012 et avec Hilaire-Pérez Liliane, Les expositions universelles. Les identités au défi de la modernité, PUR, Collection Carnot, Rennes, 2014. Contact : christiane.demeulenaere@gmail.com 